Symmetries, Differential Equations and Applications (SDEA-II)

International Journal of Modern Physics: Conference Series

Vol. 38 (2015) 1560078 (20 pages)

(C) The Author

DOI: $10.1142 / \mathrm{S} 2010194515600782$

\title{
Geometrization of Lie and Noether symmetries and applications
}

\author{
Michael Tsamparlis \\ Faculty of Physics, Department of Astrophysics - Astronomy - Mechanics \\ University of Athens, Panepistemiopolis, Athens 157 83, Greece \\ mtsampa@phys.uoa.gr \\ Published 30 April 2015
}

\begin{abstract}
We derive the Lie and the Noether conditions for the equations of motion of a dynamical system in an $n$-dimensional Riemannian space. We solve these conditions in the sense that we express the symmetry generating vectors in terms of the collineation vectors of the space. More specifically we give two theorems which contain all the necessary conditions which allow one to determine the Lie and the Noether point symmetries of a specific dynamical system evolving in a given Riemannian space in terms of the projective and the homothetic collineations of space. We apply these theorems to various interesting situations in Newtonian Physics.
\end{abstract}

\section{Introduction}

In theoretical Physics one has two main tools to study the properties of evolution of dynamical systems: (a) Symmetries of the equations of motion; and (b) Collineations (symmetries) of the background space where evolution takes place. It is well known that both these tools have the following common characteristics:

a. They form a Lie algebra.

b. They do not fix uniquely either the dynamical system or the space.

The natural question to be to asked is if these two algebras are related and if so in what way. Equivalently, one may state the question as follows:

To what degree and how does space modulate the evolution of dynamical systems in it? Is a dynamical system free to evolve at will in a given space or is it constrained to do so by the very symmetry structure of space?

This question was answered many years ago by the Theory of Relativity with the Equivalence Principle, that is, the requirement that free motion in a given gravitational field occurs along the geodesics of space. However as obvious as this

This is an Open Access article published by World Scientific Publishing Company. It is distributed under the terms of the Creative Commons Attribution 3.0 (CC-BY) License. Further distribution of this work is permitted, provided the original work is properly cited. 
point of view may appear to be it is not easy to comprehend and accept! So let us take a second more mathematical view of the situation ${ }^{\mathrm{a}}$.

In a Riemannian space the affinely parameterized geodesics are determined uniquely by the metric. The geodesics are a set of homogeneous ordinary differential equations (ODE) linear in the highest order term and quadratically non-linear in the first order terms. A system of such ODEs is characterized (not fully) by its Lie point symmetries. On the other hand a metric is characterized (again not fully) by its collineations. Therefore it is reasonable to expect that the Lie point symmetries of the system of geodesic equations of a metric will be closely related to the collineations of the metric. That such a relation exists is easy to see by the following simple example. Consider a family of straight lines parallel to the $x$-axis on the Euclidian plane. These curves can be considered either as the integral curves of the ODE $\frac{d^{2} y}{d x^{2}}=0$ or as the geodesics of the Euclidian metric $d x^{2}+d y^{2}$. Subsequently consider a symmetry operation defined by a reshuffling of these lines without preserving necessarily their parametrization. According to the first interpretation this symmetry operation is a Lie symmetry of the ODE $\frac{d^{2} y}{d x^{2}}=0$ and according to the second interpretation it is a (special) projective symmetry of the Euclidian two dimensional space.

What has been said for a Riemannian space can be generalized to a space in which there is only a linear connection. In this case the geodesics are called autoparallels (or paths) and they comprise again a system of ODEs linear in the highest order term and quadratically non-linear in the first order terms. In this case one is looking for relations between the Lie symmetries of the autoparallels and the projective collineations of the connection.

A Lie point symmetry of an ordinary differential equation (ODE) is a point transformation in the space of variables which preserves the set of solutions of the ODE. ${ }^{1-3}$ Regarding these solutions as curves in the space of variables, then we may equivalently consider a Lie point symmetry as a point transformation which preserves the set of the solution curves. Applying this observation to the geodesic curves in a Riemannian (affine) space, we infer that the Lie point symmetries of the geodesic equations in any Riemannian space are the automorphisms which preserve the set of these curves. However we know from Differential Geometry that the point transformations of a Riemannian (affine) space which preserve the set of geodesics are the projective transformations. Therefore it is reasonable to expect a correspondence between the Lie point symmetries of the geodesic equations and the projective algebra of the metric defining the geodesics.

The equation of geodesics in an arbitrary coordinate frame is a second order ODE of the form

$$
\ddot{x}^{i}+\Gamma_{j k}^{i} \dot{x}^{j} \dot{x}^{k}+F\left(x^{i}, \dot{x}^{j}\right)=0
$$

\footnotetext{
aThis point has been raised in an illuminating discussion with Prof P G Leach a few years back in Athens while we were driving to the Poseidon Temple in cape Sounion.
} 
where $F\left(x^{i}, \dot{x}^{j}\right)$ is an arbitrary function of its argument and the functions $\Gamma_{j k}^{i}$ are the connection coefficients of the space. The dot indicates differentiation with respect to the geodesic affine parameter. Equivalently equation (1) is also the equation of motion of a dynamical system moving in a Riemannian (affine) space under the action of a velocity dependent force. According to the above argument we expect that the Lie point symmetries of the ODE (1) for a given function $F\left(x^{i}, \dot{x}^{j}\right)$ will be related with the collineations of the metric. In the following we assume that $F$ is a function of $x^{i}$ only. As it will be shown in this case the Lie symmetries of (1) are a subalgebra of the special projective algebra of the space. The specific subalgebra is selected by means of certain constraint conditions involving geometric quantities of the space and the function $F\left(x^{i}, \dot{x}^{j}\right) \cdot{ }^{4-8}$

The determination of the Lie point symmetries of a given system of ODEs consists of two steps: (a) the determination of the conditions which the components of the Lie symmetry vector must satisfy; and (b) the solution of the system of these conditions. Step (a) is formal and is outlined in e.g.. ${ }^{1-3}$ The second step is the key one and, for example, in higher dimensions where one has a large number of simultaneous equations the solution can be quite involved and perhaps prohibitive. However if one expresses the system of Lie symmetry conditions of (1) in terms of collineation (i.e. symmetry) conditions of the metric, then the determination of Lie point symmetries is transferred to the geometric problem of determining the special projective group of the metric. In this field there is a significant amount of work from Differential Geometry waiting to be used. Indeed the projective symmetries are already known for many spaces or they can be determined by existing general theorems. For example the projective algebra and all its subalgebras are known for the important case of spaces of constant curvature ${ }^{12}$ and in particular for the flat spaces. This implies that, for example, the Lie symmetries of all Newtonian dynamical systems as well as those of Special Relativity can be determined by simple differentiation from the known projective algebra of these spaces!

What has been said for the Lie point symmetries of (1) applies also to Noether point symmetries (provided (1) follows from a Lagrangian). The Noether point symmetries are Lie point symmetries which satisfy the additional constraint

$$
X^{[1]} L+L \frac{d \xi}{d t}=\frac{d f}{d t} .
$$

The Noether point symmetries form a closed subalgebra of the Lie point symmetries algebra. In accordance to the above this implies that the Noether point symmetries will be related with a subalgebra of the special projection algebra of the space where 'motion' occurs. As it will be shown this subalgebra is the homothetic algebra of the space. It is well known that to each Noether point symmetry it is associated a conserved current (i.e. a Noether first integral). This leads us to the important conclusion that the (standard) conserved quantities of a dynamical system depend on the space in which it moves and the type of force $F\left(x^{i}\right)$ which modulates the motion. In particular in 'free fall', that is when $F\left(x^{i},\right)=0$ the orbits are affinely 
parametrized geodesics and the geometry of the space is the sole factor which determines the conserved quantities of motion. This conclusion is by no means trivial and means that the space where motion occurs is not a pathetic carrier of motion but it is the major modulator of the evolution of a dynamical system. In other words there is a strong and deep relation between Geometry of the space and Physics (motion) in that space!

In what follows we discuss the aforementioned ideas in a systematic way. More specifically we do the following

a. We derive the general expression for the Lie symmetry conditions of (1) assuming a general (i.e. not necessarily symmetric) connection in terms of geometric quantities;

b. For the case that the function $F$ depends only on the coordinates, i.e. $F\left(x^{i}\right)$ we give two theorems which establish the exact relation between the (special) projective/homothetic algebra of the space and the Lie/Noether symmetry algebra of (1) respectively;

c. We consider applications in Newtonian Physics;

d. We use the above general results to generalize the Ermakov- Pinney dynamical system to $n$ dimensional Riemannian space.

\section{Collineations of Riemannian Spaces}

For convenience we give a brief account of the basic definitions concerning collineations in a Riemannian space. A collineation in a Riemannian space is a vector field $\mathbf{X}$ which satisfies an equation of the form

$$
\mathcal{L}_{X} \mathbf{A}=\mathbf{B}
$$

where $\mathcal{L}_{X}$ denotes Lie derivative, $\mathbf{A}$ is a geometric object (not necessarily a tensor) defined in terms of the metric and its derivatives (e.g. connection, Ricci tensor, curvature tensor etc.) and $\mathbf{B}$ is an arbitrary tensor with the same tensor indices as A. The collineations in a Riemannian space have been classified by Katzin et al.. ${ }^{10}$ In the following we use only certain collineations.

A conformal Killing vector $(\mathrm{CKV})$ is defined by the relation

$$
\mathcal{L}_{X} g_{i j}=2 \psi\left(x^{k}\right) g_{i j}
$$

If $\psi=0, \mathbf{X}$ is called a Killing vector $(\mathrm{KV})$, if $\psi$ is a non-vanishing constant a homothetic vector $(\mathrm{HV})$ and if $\psi_{; i j}=0$, a special conformal Killing vector (SCKV). A CKV is called proper if it is not a $\mathrm{KV}, \mathrm{HV}$ or a SCKV.

A projective collineation (PC) is defined by the equation

$$
\mathcal{L}_{X} \Gamma_{j k}^{i}=2 \phi_{(, j} \delta_{k)}^{i}
$$

If $\phi=0$ the $\mathrm{PC}$ is called an affine collineation (AC) and if $\phi_{; i j}=0$ a special projective collineation ( $\mathrm{SPC}$ ). A proper $\mathrm{PC}$ is a $\mathrm{PC}$ which is not an $\mathrm{AC}, \mathrm{HV}$ or $\mathrm{KV}$ or SPC. The PCs form a Lie algebra whose ACs, HV and KVs form subalgebras. It 


\begin{tabular}{|l|c|l|}
\hline Collineation & A & B \\
\hline Killing vector (KV) & $g_{i j}$ & 0 \\
\hline Homothetic vector (HV) & $g_{i j}$ & $\psi g_{i j}, \psi_{, i}=0$ \\
\hline Conformal Killing vector (CKV) & $g_{i j}$ & $\psi g_{i j}, \psi,,_{i} \neq 0$ \\
\hline Affine Collineation (AC) & $\Gamma_{j k}^{i}$ & 0 \\
\hline Projective collineation (PC) & $\Gamma_{j k}^{i}$ & $2 \phi_{(, j} \delta_{k)}^{i}, \phi,{ }_{i} \neq 0$ \\
\hline Special Projective collineation (SPC) & $\Gamma_{j k}^{i}$ & $2 \phi_{(, j} \delta_{k)}^{i}, \phi,,_{i} \neq 0$ and $\phi,{ }_{j k}=0$ \\
\hline
\end{tabular}

\begin{tabular}{|l|l|l|}
\hline Collineation & Gradient & Non-gradient \\
\hline Killing vectors (KV) & $\mathbf{S}_{I}=\delta_{I}^{i} \partial_{i}$ & $\mathbf{X}_{I J}=\delta_{[I}^{j} \delta_{j]}^{i} x_{j} \partial_{i}$ \\
\hline Homothetic vector (HV) & $\mathbf{H}=x^{i} \partial_{i}$ & \\
\hline Affine Collineation (AC) & $\mathbf{A}_{I J}=x_{J} \delta_{I}^{i} \partial_{i}$ & \\
\hline Special Projective collineation (SPC) & & $\mathbf{P}_{I}=S_{I} \mathbf{H}$. \\
\hline
\end{tabular}

has been shown that if a metric admits a SCKV then also admits a SPC, a gradient $\mathrm{HV}$ and a gradient KV. ${ }^{11}$ We summarize the above in Table 1.

In the following we shall need the symmetry algebra of spaces of constant curvature. $\mathrm{In}^{12}$ it has been shown that the PCs of a space of constant non-vanishing curvature consist of proper PCs and KVs only and if the space is flat then the algebra of the PCs consists of KVs/HV/ACs and SPCs. For convenience we summarize these results in Table 2.

The Lie point symmetries of all Newtonian dynamical systems are amongst the vectors in Table 2. Also the Noether point symmetries of all Newtonian dynamical systems (and more generally all systems moving in a flat space) follow from the elements of the first two rows of Table 2 (apart form some differences in sign depending on the signature of the metric).

\section{The Lie Point Point Symmetry Conditions in an Affine Space}

We consider the system of ODEs:

$$
\ddot{x}^{i}+\Gamma_{j k}^{i} \dot{x}^{j} \dot{x}^{k}+\sum_{m=0}^{n} P_{j_{1} \ldots j_{m}}^{i} \dot{x}^{j_{1}} \ldots \dot{x}^{j_{m}}=0
$$

where $\Gamma_{j k}^{i}$ are the connection coefficients of the space and $P_{j_{1} \ldots j_{m}}^{i}\left(t, x^{i}\right)$ are smooth polynomials completely symmetric in the lower indices and derive the Lie point symmetry conditions in geometric form using the standard approach. Equation (6) is quite general and covers most of the standard autonomous and non autonomous dynamical systems and in particular equation (1). Furthermore because the $\Gamma_{j k}^{i}$ 's are not assumed to be symmetric, the results are valid in a space with torsion. Obviously they hold in a Riemannian space where the connection coefficients are given in terms of the Christofell symbols. 


\section{Tsamparlis}

The detailed calculation can be found $\mathrm{in}^{8}$ and shall not be repeated here. In the following we summarize the results.

The terms $\dot{x}^{j_{1}} \ldots \dot{x}^{j_{m}}$ for $m \leq 4$ give the equations:

$$
\begin{aligned}
& L_{\eta} P^{i}+2 \xi,{ }_{t} P^{i}+\xi P^{i}{ }_{t}+\eta^{i},{ }_{t t}+\eta^{j}{ }_{, t} P_{. j}^{i}=0 \\
& L_{\eta} P_{j}^{i}+\xi{ }_{t} P_{j}^{i}+\xi P_{j}^{i}, t+\left(\xi,{ }_{k} \delta_{j}^{i}+2 \xi,{ }_{j} \delta_{k}^{i}\right) P^{k}+2 \eta^{i},{ }_{t \mid j}-\xi{ }_{, t} \delta_{k}^{i}+2 \eta^{k}{ }_{t} P_{. j k}^{i}=0 \\
& L_{\eta} P_{j k}^{i}+L_{\eta} \Gamma_{j k}^{i}+\left(\xi,_{d} \delta_{(k}^{i}+\xi,{ }_{(k} \delta_{|d|}^{i}\right) P_{. j)}^{d}+\xi P_{. k j, t}^{i}-2 \xi_{, t(j} \delta_{k)}^{i}+3 \eta^{d}{ }_{, t} P_{. d k j}^{i}=0 \\
& L_{\eta} P_{. j k d}^{i}-\xi,_{t} P_{. j k d}^{i}+\xi,_{e} \delta_{(k}^{i} P_{. d j)}^{e}+\xi P_{. j k d, t}^{i}+4 \eta^{e}{ }_{t} P_{. j k d e}^{i}-\xi_{(, j \mid k} \delta_{d)}^{i}=0
\end{aligned}
$$

and the conditions due to the terms $\dot{x}^{j_{1}} \ldots \dot{x}^{j_{m}}$ for $m>4$ are given by the following general formula:

$$
\begin{aligned}
& L_{\eta} P_{j_{1} \ldots j_{m}}^{i}+P_{j_{1} \ldots j_{m}, t}^{i} \xi+(2-m) \xi_{, t} P_{j_{1} \ldots j_{m}}^{i}+\xi_{, r}(2-(m-1)) P_{j_{1} \ldots j_{m-1}}^{i} \delta_{j_{m}}^{r} \\
& \quad+(m+1) P_{j_{1} \ldots j_{m+1}}^{i} \eta_{, t}^{j_{m+1}}+\xi_{, j} P_{j_{1} \ldots j_{m-1}}^{j} \delta_{j_{m}}^{i}=0 .
\end{aligned}
$$

Note the appearance of the term $L_{\eta} \Gamma_{j k}^{i}$ in these expressions.

Eqn. (1) is obtained for $m=0, P^{i}=F^{i}$ in which case the Lie symmetry conditions read:

$$
\begin{aligned}
& L_{\eta} P^{i}+2 \xi_{, t} P^{i}+\xi P^{i},{ }_{t}+\eta^{i},{ }_{t t}=0 \\
& \left(\xi_{, k} \delta_{j}^{i}+2 \xi,_{j} \delta_{k}^{i}\right) P^{k}+2 \eta^{i},{ }_{t \mid j}-\xi,{ }_{, t} \delta_{k}^{i}=0 \\
& L_{\eta} \Gamma_{j k}^{i}-2 \xi,_{t(j} \delta_{k)}^{i}=0 \\
& \xi_{(, j \mid k} \delta_{d)}^{i}=0 .
\end{aligned}
$$

If $F^{i}=0$ we obtain the Lie symmetry conditions for the geodesic equations (see ${ }^{8}$ and $\left.^{9}\right)$.

\section{The Autonomous Dynamical System Moving in a Riemannian Space}

We 'solve' the Lie symmetry conditions (12) - (15) for an autonomous dynamical system in the sense that we express them in terms of the collineations of the metric of the space where motion occurs.

Equation (15) means that $\xi_{, j}$ is a gradient $\mathrm{KV}$ of $g_{i j}$. This implies that the metric $g_{i j}$ is decomposable. Equation (14) means that $\eta^{i}$ is a projective collineation of the metric with projective function $\xi_{, t}$. The remaining two equations are constraint conditions, which relate the components $\xi, n^{i}$ of the Lie symmetry vector with the vector $F^{i}\left(x^{j}\right)$. Equation (12) gives

$$
\left(L_{\eta} g^{i j}\right) F_{j}+g^{i j} L_{\eta} F_{j}+2 \xi_{, t} g^{i j} F_{j}+\eta_{, t t}^{i}=0 .
$$


This equation is an additional restriction for $\eta^{i}$ because it relates it directly to the symmetries of the metric. Finally equation (13) gives

$$
-\delta_{j}^{i} \xi_{, t t}+\left(\xi_{, j} \delta_{k}^{i}+2 \delta_{j}^{i} \xi_{, k}\right) F^{k}+2 \eta_{, t j}^{i}+2 \Gamma_{j k}^{i} \eta_{, t}^{k}=0
$$

We conclude that the Lie symmetry equations is given by equations $(16),(17)$ where $\xi(t, x)$ is a gradient $\mathrm{KV}$ of the metric and $\eta^{i}(t, x)$ is a special projective collineation of the metric with projective function $\xi_{, t}$. We state this result in theorem 4.1 below. $^{8}$

Theorem 4.1. The Lie point symmetries of the system of equations of motion of an autonomous system under the action of the force $F^{j}\left(x^{i}\right)$ in a general Riemannian space with metric $g_{i j}$, namely

$$
\ddot{x}^{i}+\Gamma_{j k}^{i} \dot{x}^{j} \dot{x}^{k}=F^{i}
$$

are given in terms of the generators $Y^{i}$ of the special projective algebra of the metric $g_{i j}$.

We consider next the case that the force $F^{i}$ is derivable from a potential $V\left(x^{i}\right)$, so that the equations of motion follow from the standard Lagrangian

$$
L\left(x^{j}, \dot{x}^{j}\right)=\frac{1}{2} g_{i j} \dot{x}^{i} \dot{x}^{j}-V\left(x^{j}\right)
$$

with Hamiltonian

$$
E=\frac{1}{2} g_{i j} \dot{x}^{i} \dot{x}^{j}+V\left(x^{j}\right)
$$

The Noether conditions are

$$
\begin{aligned}
V_{, k} \eta^{k}+V \xi_{, t} & =-f_{, t} \\
\eta_{, t}^{i} g_{i j}-\xi_{, j} V & =f_{, j} \\
L_{\eta} g_{i j} & =2\left(\frac{1}{2} \xi_{, t}\right) g_{i j} \\
\xi_{, k} & =0 .
\end{aligned}
$$

Thew last equation implies $\xi=\xi(t)$ and reduces the system as follows:

$$
\begin{aligned}
L_{\eta} g_{i j} & =2\left(\frac{1}{2} \xi_{, t}\right) g_{i j}, \\
V_{, k} \eta^{k}+V \xi_{, t} & =-f_{, t} \\
\eta_{i, t} & =f_{, i} .
\end{aligned}
$$

Equation (25) implies that $\eta^{i}$ is a conformal Killing vector of the metric provided $\xi_{, t} \neq 0$. Because $g_{i j}$ is independent of $t$ and $\xi=\xi(t)$ the $\eta^{i}$ must be is a HV of the metric. This means that $\eta^{i}(t, x)=T(t) Y^{i}\left(x^{j}\right)$ where $Y^{i}$ is a HV. If $\xi_{, t}=0$ then $\eta^{i}$ is a Killing vector of the metric. Equations (26), (27) are the constraint conditions, which the Noether symmetry and the potential must satisfy for the former to be admitted. The above lead to the following theorem. ${ }^{8}$ 
Theorem 4.2. The Noether point symmetries of the Lagrangian (19) are generated from the homothetic algebra of the metric $g_{i j}$.

More specifically, concerning the Noether point symmetries, we have the following.

All autonomous systems admit the Noether symmetry $\partial_{t}$ whose Noether integral is the Hamiltonian $E$ given in equation (20). For the rest of the Noether point symmetries we have the following cases

Case I Noether point symmetries generated by the homothetic algebra.

The Noether symmetry vector and the Noether function $G\left(t, x^{k}\right)$ are

$$
\mathbf{X}=2 \psi_{Y} t \partial_{t}+Y^{i} \partial_{i}, G\left(t, x^{k}\right)=p t
$$

where $\psi_{Y}$ is the homothety factor of $Y^{i}\left(\psi_{Y}=0\right.$ for a KV and 1 for the HV), and $p$ is a constant, provided the potential satisfies the condition

$$
\mathcal{L}_{Y} V+2 \psi_{Y} V+p=0 .
$$

Case II Noether point symmetries generated by the gradient homothetic Lie algebra i.e. both KVs and the HV must be gradient.

In this case the Noether symmetry vector and the Noether function are

$$
\mathbf{X}=2 \psi_{Y} \int T(t) d t \partial_{t}+T(t) H^{i} \partial_{i}, G\left(t, x^{k}\right)=T_{, t} H\left(x^{k}\right)+p \int T d t,
$$

where $H^{i}$ is the gradient $\mathrm{HV}$ or a gradient $\mathrm{KV}$, the function $T(t)$ is computed from the relation $T_{, t t}=m T$ where $m$ is a constant and the potential satisfies the condition

$$
\mathcal{L}_{H} V+2 \psi_{Y} V+m H+p=0 .
$$

Concerning the Noether integrals we have the following result (not including the Hamiltonian)

Corollary 4.1. The Noether integrals of Case I and Case II are respectively

$$
\begin{aligned}
& I_{C_{I}}=2 \psi_{Y} t E-g_{i j} Y^{i} \dot{x}^{j}+p t \\
& I_{C_{I I}}=2 \psi_{Y} \int T(t) d t E-g_{i j} H^{, i} \dot{x}^{j}+T_{, t} H+p \int T d t .
\end{aligned}
$$

where $E$ is the Hamiltonian given in (20).

We remark that theorems 4.1 and 4.2 do not apply to generalized or dynamical symmetries.

Let us see a first application of the above general theorems.

\section{The Lie Point Symmetries of Geodesic Equations in an Einstein Space}

Spaces of constant curvature are Einstein spaces whose curvature tensor is of the form $R_{a b c d}=\frac{R}{n(n-1)}\left(g_{a c} g_{b d}-g_{a d} g_{b c}\right)$ where $R$ is a constant. A proper Einstein 
space is a space in which $R_{a b}=\frac{R}{n} g_{a b}$ but ${ }^{\mathrm{b}} R_{a b c d} \neq \frac{R}{n(n-1)}\left(g_{a c} g_{b d}-g_{a d} g_{b c}\right)$. The projective collineations of Einstein spaces can be found in. ${ }^{12}$

Suppose $X^{a}$ is a projective collineation with projection function $\phi\left(x^{a}\right)$ so that $\mathcal{L}_{X} \Gamma_{b c}^{a}=\phi_{, b} \delta_{c}^{a}+\phi_{, c} \delta_{b}^{a}$. For a proper Einstein space :

$$
\mathcal{L}_{X} g_{a b}=\frac{n(1-n)}{R} \phi_{; a b}
$$

It follows that if $X^{a}$ generates either an affine or a special projective collineation then $\phi_{; a b}=0$ hence $X^{a}$ reduces to a KV. This means that proper Einstein spaces do not admit a homothetic vector, affine collineations or special projective collineations.

The above results and Theorem 4.1 lead to the following conclusion:

Theorem 5.1. The Lie point symmetries of the geodesic equations in a proper Einstein space of curvature scalar $R(\neq 0)$ are given by the vectors

$$
\mathbf{X}=(K t+L) \partial_{t}+D^{i}(x) \partial_{x^{i}},
$$

where $D^{i}(x)$ is a $K V$ of the metric and $K, L$ are constants.

Theorem 5.1 extends and amends the conjecture of ${ }^{13}$ to the more general case of Einstein spaces.

\section{Applications in Newtonian Physics}

One important problem to consider is to find all 2-d and 3 -d dimensional autonomous Newtonian dynamical systems, that is, all dynamical systems moving in flat Euclidian space with equation of motion $\ddot{x}^{i}=F^{i}\left(x^{j}\right)$ where $i, j=1,2$ or 1, 2, 3 respectively which admit Lie and Noether point symmetries and of course to determine the admitted symmetries. The answer to this question consists of finding all the forces $F^{i}\left(x^{j}\right)$ which result in this property.

The importance of this problem is twofold. Indeed the knowledge of the Lie symmetries makes possible the computation of the invariants of the dynamical system; also the knowledge of Noether symmetries gives the Noether integrals (conserved currents) of the system. Both these data can be used to simplify the equations of motion and even lead to their analytic solution.

The two dimensional case has been considered originally in ${ }^{14}$ and the $3 \mathrm{~d}$ case in. ${ }^{15,16}$ Both cases have been considered anew following the geometric approach of the previous paragraphs. More specifically the 2 -d case has been considered in ${ }^{17}$ and the $3 \mathrm{~d}$ case in. ${ }^{18}$ In both cases it has been shown that the existing results were incomplete.

It is to be noted that from the applications point of view the $2 \mathrm{~d}$ case is important because it applies to the mini super space which is particularly useful in cosmology.

\footnotetext{
${ }^{\mathrm{b}}$ Using the contracted Bianchi identity $\left(R^{i j}-\frac{1}{2} R g^{i j}\right)_{; j}=0$ it follows that in an Einstein space of dimension $n>2$ the curvature scalar $R=$ constant.
} 


\begin{tabular}{|l|l|l|l|}
\hline Lie symmetry & $\mathbf{F}_{\mu}\left(x_{\mu}, x_{\nu}, x_{\sigma}\right)$ & $\mathbf{F}_{\nu}\left(x_{\mu}, x_{\nu}, x_{\sigma}\right)$ & $\mathbf{F}_{\sigma}\left(x_{\mu}, x_{\nu}, x_{\sigma}\right)$ \\
\hline$\frac{d}{2} t \partial_{t}+\partial_{\mu}$ & $e^{-d x_{\mu}} f\left(x_{\nu}, x_{\sigma}\right)$ & $e^{-d x_{\mu}} g\left(x_{\nu}, x_{\sigma}\right)$ & $e^{-d x_{\mu} h\left(x_{\nu}, x_{\sigma}\right)}$ \\
\hline$\frac{d}{2} t \partial_{t}+\partial_{\theta}(\mu \nu)$ & $e^{-d \theta(\mu \nu} f\left(r_{(\mu \nu)}, x_{\sigma}\right)$ & $e^{-d \theta(\mu \nu)} g\left(r_{(\mu \nu)}, x_{\sigma}\right)$ & $e^{-d \theta(\mu \nu)} h\left(r_{(\mu \nu)}, x_{\sigma}\right)$ \\
\hline$\frac{d}{2} t \partial_{t}+R \partial_{R}$ & $x_{\mu}^{1-d} f\left(\frac{x_{\nu}}{x_{\mu}}, \frac{x_{\sigma}}{x_{\mu}}\right)$ & $x_{\mu}^{1-d} g\left(\frac{x_{\nu}}{x_{\mu}}, \frac{x_{\sigma}}{x_{\mu}}\right)$ & $x_{\mu}^{1-d} h\left(\frac{x_{\nu}}{x_{\mu}}, \frac{x_{\sigma}}{x_{\mu}}\right)$ \\
\hline$\frac{d}{2} t \partial_{t}+x_{\mu} \partial_{\mu}$ & $x_{\mu}^{1-d} f\left(x_{\nu}, x_{\sigma}\right)$ & $x_{\mu}^{1-d} g\left(x_{\nu}, x_{\sigma}\right)$ & $x_{\mu}^{1-d} h\left(x_{\nu}, x_{\sigma}\right)$ \\
\hline$\frac{d}{2} t \partial_{t}+x_{\nu} \partial_{\mu}$ & $\begin{array}{r}e^{-d \frac{x \mu}{x_{\nu}}}\left[\frac{x_{\mu}}{x_{\nu}} g\left(x_{\nu}, x_{\sigma}\right)\right. \\
\left.\quad+f\left(x_{\nu}, x_{\sigma}\right)\right]\end{array}$ & $e^{-d \frac{x_{\mu}}{x_{\nu}}} g\left(x_{\nu}, x_{\sigma}\right)$ & $e^{-d \frac{x_{\mu}}{x_{\nu}} h\left(x_{\nu}, x_{\sigma}\right)}$ \\
\hline
\end{tabular}

\begin{tabular}{|c|c|c|c|}
\hline Lie symmetry & $\mathbf{F}_{\mu}\left(x_{\mu}, x_{\nu}, x_{\sigma}\right)$ & $\mathbf{F}_{\nu}\left(x_{\mu}, x_{\nu}, x_{\sigma}\right)$ & $\mathbf{F}_{\sigma}\left(x_{\mu}, x_{\nu}, x_{\sigma}\right)$ \\
\hline$t \partial_{\mu}$ & $f\left(x_{\nu}, x_{\sigma}\right)$ & $g\left(x_{\nu}, x_{\sigma}\right)$ & $h\left(x_{\nu}, x_{\sigma}\right)$ \\
\hline$t^{2} \partial_{t}+t R \partial_{R}$ & $\frac{1}{x_{\mu}^{3}} f\left(\frac{x_{\nu}}{x_{\mu}}, \frac{x_{\sigma}}{x_{\mu}}\right)$ & $\frac{1}{x_{\mu}^{3}} g\left(\frac{x_{\nu}}{x_{\mu}}, \frac{x_{\sigma}}{x_{\mu}}\right)$ & $\frac{1}{x_{\mu}^{3}} h\left(\frac{x_{\nu}}{x_{\mu}}, \frac{x_{\sigma}}{x_{\mu}}\right)$ \\
\hline$e^{ \pm t \sqrt{m}} \partial_{\mu}$ & $-m x_{\mu}+f\left(x_{\nu}, x_{\sigma}\right)$ & $g\left(x_{\nu}, x_{\sigma}\right)$ & $h\left(x_{\nu}, x_{\sigma}\right)$ \\
\hline $\begin{array}{l}\frac{1}{\sqrt{m}} e^{ \pm t \sqrt{m}} \partial_{t} \\
+e^{ \pm t \sqrt{m}} R \partial_{R}\end{array}$ & $\begin{array}{l}-\frac{m}{4} x_{\mu} \\
\quad+\frac{1}{x_{\mu}^{3}} f\left(\frac{x_{\nu}}{x_{\mu}}, \frac{x_{\sigma}}{x_{\mu}}\right)\end{array}$ & $\begin{aligned}- & \frac{m}{4} x_{\nu} \\
& \quad+\frac{1}{x_{\mu}^{3}} g\left(\frac{x_{\nu}}{x_{\mu}}, \frac{x_{\sigma}}{x_{\mu}}\right)\end{aligned}$ & $-\frac{m}{4} x_{\sigma}+\frac{1}{x_{\mu}^{3}} h\left(\frac{x_{\nu}}{x_{\mu}}, \frac{x_{\sigma}}{x_{\mu}}\right)$ \\
\hline
\end{tabular}

Indeed in the standard cosmological model and many of its extensions the resulting dynamical system reduces to a two dimensional dynamical system in a flat 2-d Lorentz space. For convenience we collect the results of the 3 -d case in Tables 3 and 4. Concerning the determination of all 3-d Newtonian dynamical systems, which admit Noether point symmetries and subsequently the ones which are integrable via Noether integrals we have the results of the Table 5 and Table 6 .

\begin{tabular}{|l|l|}
\hline Noether Symmetry & $\mathbf{V}(\mathbf{x}, \mathbf{y}, \mathbf{z}) / \mathbf{T}_{, t t}=\mathbf{m} \mathbf{T}$ \\
\hline$\partial_{\mu}$ & $-p x_{\mu}+f\left(x^{\nu}, x^{\sigma}\right)$ \\
\hline$x_{\nu} \partial_{\mu}-x_{\mu} \partial_{\nu}$ & $-p \theta_{(\mu \nu)}+f\left(r_{(\mu \nu)}, x^{\sigma}\right)$ \\
\hline $2 t \partial_{t}+R \partial_{R}$ & $\frac{1}{R^{2}} f(\theta, \phi)$ or $\frac{1}{x_{\mu}^{2}} f\left(\frac{x_{\nu}}{x_{\mu}}, \frac{x_{\sigma}}{x_{\mu}}\right)$ \\
\hline$T(t) \partial_{\mu}$ & $-\frac{m}{2} x_{\mu}^{2}-p x_{\mu}+f\left(x_{\nu}, x_{\sigma}\right)$ \\
\hline$\left(2 \int T(t) d t\right) \partial_{t}+T(t) R \partial_{R}$ & $-\frac{m}{8} R^{2}+\frac{1}{R^{2}} f(\theta, \phi)$ or $-\frac{m}{8} R^{2}+\frac{1}{x_{\mu}^{2}} f\left(\frac{x_{\nu}}{x_{\mu}}, \frac{x_{\sigma}}{x_{\mu}}\right)$ \\
\hline
\end{tabular}

\begin{tabular}{|l|l|}
\hline Noether Symmetry & $\mathbf{V}(\mathbf{x}, \mathbf{y}, \mathbf{z})$ \\
\hline$a \partial_{\mu}+b \partial_{\nu}$ & $-\frac{p}{a} x_{\mu}+f\left(x^{\nu}-\frac{b}{a} x^{\mu}, x^{\sigma}\right)$ \\
\hline$a \partial_{\mu}+b\left(x_{\nu} \partial_{\mu}-x_{\mu} \partial_{\nu}\right)$ & $-\frac{p}{|b|} \arctan \left(\frac{|b| x_{\mu}}{\left|\left(a+b x_{\nu}\right)\right|}\right)+f\left(\frac{1}{2} r_{(\mu \nu)}+\frac{a}{b} x^{\nu}, x^{\sigma}\right)$ \\
\hline$a \partial_{\mu}+b\left(x_{\sigma} \partial_{\nu}-x_{\nu} \partial_{\sigma}\right)$ & $-\frac{p}{|b|} \theta_{(\nu \sigma)}+f\left(r_{(\nu \sigma)}, x^{\mu}-\frac{a}{b} \theta_{(\nu \sigma)}\right)$ \\
\hline$a\left(x_{\nu} \partial_{\mu}-x_{\mu} \partial_{\nu}\right)+$ & $\frac{p}{a} \arctan \left(\frac{a x_{\nu}+b x_{\sigma}}{x_{\mu} \sqrt{a^{2}+b^{2}}}\right)+$ \\
& $+b\left(x_{\sigma} \partial_{\mu}-x_{\mu} \partial_{\sigma}\right)$ \\
\hline $2 b t \partial_{t}+a \partial_{\mu}+b R \partial_{R}$ & $+\frac{1}{a} f\left(x_{\sigma}-\frac{a}{b} x_{\nu}, x_{\nu}^{2}\left(1-\left(\frac{a}{b}\right)^{2}+\frac{2 b}{a} \frac{x_{\sigma}}{x_{\nu}}\right)+x_{\mu}^{2}\right)$ \\
\hline $2 b t \partial_{t}+a \theta_{(\mu \nu)} \partial_{\theta_{(\mu \nu)}}+b R \partial_{R}$ & $\frac{1}{2\left(a+b x_{\mu}^{2}\right)}+\frac{1}{\left(a+b x_{\mu}^{2}\right)} f\left(\frac{x_{\nu}}{a+b x_{\mu}}, \frac{x_{\sigma}}{a+b x_{\mu}}\right)$ \\
\hline
\end{tabular}


In order to show the use of these tables we consider the dynamical system defined by the equations of motion

$$
\ddot{x}^{\mu}=-\frac{m}{4} x_{\mu}+\frac{1}{x_{\mu}^{3}} f_{\mu, \nu, \sigma}\left(\frac{x_{\nu}}{x_{\mu}}, \frac{x_{\sigma}}{x_{\mu}}\right)
$$

This system is the well known Ermakov system which has a long history in the literature. ${ }^{19-23}$

From Table 5 we find without any calculations that this system admits the Lie point symmetry $\mathbf{X}=\frac{1}{\sqrt{m}} e^{ \pm t \sqrt{m}} \partial_{t}+e^{ \pm t \sqrt{m}} R \partial_{R}$.

In the following section we combine all the above results in order to generalize the Ermakov dynamical system.

\section{The Ermakov Dynamical System}

The Ermakov system has its roots in the study of the one dimensional time dependent harmonic oscillator

$$
\ddot{x}+\omega^{2}(t) x=0 .
$$

Ermakov $^{24}$ obtained a first integral $J$ of this equation by introducing the auxiliary equation

$$
\ddot{\rho}+\omega^{2}(t) \rho=\rho^{-3}
$$

eliminating the $\omega^{2}(t)$ term and multiplying with the integrating factor $\rho \dot{x}-\dot{\rho} x$ :

$$
J=\frac{1}{2}\left[(\rho \dot{x}-\dot{\rho} x)^{2}+(x / \rho)^{2}\right] .
$$

A short review of these studies and a detailed list of relevant references can be found in. ${ }^{25}$ Earlier reviews of the Ermakov system and its numerous applications in divertive areas of Physics can be found in, ${ }^{26} .{ }^{27}$

The general Ermakov system does not admit Lie point symmetries. The form of the most general Ermakov system which admits Lie point symmetries has been determined in ${ }^{28}$ and it is called the Kepler - Ermakov system ${ }^{29} \cdot{ }^{30}$ It is well known that these Lie symmetries are a representation of the $\operatorname{sl}(2, R)$ algebra.

In an attempt to generalize the Kepler - Ermakov system to higher dimensions Leach $^{30}$ used the $\operatorname{sl}(2, R)$ invariance of the two dimensional (Euclidean) Kepler Ermakov system and demanded that the 'generalized' Kepler - Ermakov system will posses two properties (a) A first integral, the Ermakov invariant; and (b) $\operatorname{sl}(2, R)$ invariance. It has been shown that the invariance group of the Ermakov invariant is reacher than $\operatorname{sl}(2, R) .{ }^{31}$ In this section we follow Leach's proposal and use the theorems of the previous sections to generalize the Kepler - Ermakov system in two directions: (a) to higher dimensions using the $s l(2, R)$ invariance with respect to Noether symmetries (provided the system is Hamiltonian); and (b) in a Riemannian space which admits a gradient homothetic vector (HV). 


\section{The Two-Dimensional Kepler Ermakov System}

The most general 2-d Newtonian Ermakov system is defined by the equations ${ }^{28}$ :

$$
\begin{aligned}
& \ddot{x}+\omega^{2}(t, x, y, \dot{x}, \dot{y}) x=\frac{1}{y x^{2}} f\left(\frac{y}{x}\right), \\
& \ddot{y}+\omega^{2}(t, x, y, \dot{x}, \dot{y}) y=\frac{1}{x y^{2}} g\left(\frac{x}{y}\right) .
\end{aligned}
$$

This system admits the Ermakov first integral

$$
I=\frac{1}{2}(x \dot{y}-y \dot{x})^{2}+\int^{y / x} f(\tau) d \tau+\int^{y / x} g(\tau) d \tau .
$$

By means of the transformation:

$$
\begin{gathered}
\Omega^{2}=\omega^{2}-\frac{1}{x y^{3}} g\left(\frac{x}{y}\right), \\
F\left(\frac{y}{x}\right)=f\left(\frac{y}{x}\right)-\frac{x^{2}}{y^{2}} g\left(\frac{x}{y}\right),
\end{gathered}
$$

equations (38) and (39) take the form:

$$
\begin{aligned}
& \ddot{x}+\Omega^{2}(x, y, \dot{x}, \dot{y}) x=\frac{1}{x^{2} y} F\left(\frac{y}{x}\right), \\
& \ddot{y}+\Omega^{2}(x, y, \dot{x}, \dot{y}) y=0 .
\end{aligned}
$$

Due to the second equation, except for special cases, the new function $\Omega$ is independent of $t$ and depends only on the dynamical variables $x, y$ and possibly on their derivative. The Ermakov first integral in the new variables is:

$$
I=\frac{1}{2}(x \dot{y}-y \dot{x})^{2}+\int^{y / x} F(\tau) d \tau .
$$

The system of equations (41), (42) defines the most general 2-d Ermakov system and produces all its known forms for special choices of the function $\Omega$. For example the weak Kepler - Ermakov system ${ }^{30}$ defined by the equations ${ }^{29}$

$$
\begin{aligned}
& \ddot{x}+\frac{x}{r^{3}} H(x, y)-\frac{1}{x^{3}} f\left(\frac{y}{x}\right)=0 \\
& \ddot{y}+\frac{y}{r^{3}} H(x, y)-\frac{1}{y^{3}} g\left(\frac{y}{x}\right)=0
\end{aligned}
$$

where $H, f, g$ are arbitrary functions of their argument, is obtained by the function

$$
\Omega^{2}(x, y)=H(x, y) / r^{3}
$$

with Ermakov first integral

$$
I=\frac{1}{2}(x \dot{y}-y \dot{x})^{2}+\int^{\frac{y}{x}}\left[\lambda f(\lambda)-\lambda^{-3} g(\lambda)\right] d \lambda .
$$

The weak Kepler Ermakov system does not admit Lie point symmetries, however the property of having a first integral prevails. 
The system of equations (44) - (45) admits Lie point symmetries only for $H(x, y)=-\mu^{2} r^{3}+\frac{h\left(\frac{y}{x}\right)}{x}$ where $\mu \in \mathbb{C}$. This is the (standard) Kepler - Ermakov system defined by the equations ${ }^{19,32}$

$$
\begin{gathered}
\ddot{x}-\mu^{2} x+\frac{1}{r^{3}} h\left(\frac{y}{x}\right)-\frac{1}{x^{3}} f\left(\frac{y}{x}\right)=0, \\
\ddot{y}-\mu^{2} y+\frac{1}{r^{3}} \frac{y}{x} h\left(\frac{y}{x}\right)-\frac{1}{y^{3}} g\left(\frac{y}{x}\right)=0 .
\end{gathered}
$$

The admitted Lie symmetry vectors are

$$
\mathbf{X}=\left(c_{1}-c_{2} \frac{1}{\mu} e^{2 \mu t}+c_{3} \frac{1}{\mu} e^{-2 \mu t}\right) \partial_{t}+\left(c_{2} e^{2 \mu t}+c_{3} e^{-2 \mu t}\right) R \partial_{R} \quad(\mu \neq 0)
$$

and

$$
X^{1}=\partial_{t}, X^{2}=2 t \partial_{t}+R \partial_{R}, X^{3}=t^{2} \partial_{t}+t R \partial_{R} \quad(\mu=0)
$$

where $R \partial_{R}=x \partial_{x}+y \partial_{y}$ is the gradient HV of the 2 -d Euclidian metric. Each set of vectors in (50) and (51) is a representation of the $\operatorname{sl}(2, R)$ algebra. It is important to note that these vectors are constructed from the vector $\partial_{t}$ and the gradient $\mathrm{HV}$ $R \partial_{R}$ of the Euclidian two dimensional space $E^{2}$.

Equations (48), (49) follow from the Lagrangian ${ }^{32}$

$$
L=\frac{1}{2}\left(\dot{r}^{2}+r^{2} \dot{\theta}^{2}\right)-\frac{\mu^{2}}{2} r^{2}-\frac{C(\theta)}{2 r^{2}},
$$

where $C(\theta)=c+\sec ^{2} \theta f(\tan \theta)+\csc ^{2} \theta g(\tan \theta)$ provided the functions $f, g$ satisfy the constraint:

$$
\sin ^{2} \theta f^{\prime}(\tan \theta)+\cos ^{2} \theta g^{\prime}(\tan \theta)=0
$$

The Ermakov invariant in this case is. ${ }^{32}$

$$
J=r^{4} \dot{\theta}^{2}+2 C(\theta) .
$$

If a Kepler - Ermakov system is Hamiltonian then the Lie symmetries are also Noether point symmetries with Noether integrals the Hamiltonian ${ }^{33}$

$$
E=\frac{1}{2}\left(\dot{r}^{2}+r^{2} \dot{\theta}^{2}\right)+\frac{1}{2} \mu^{2} r^{2}+\frac{1}{r^{2}} F(\theta)
$$

and two additional time dependent Noether integrals as follows: $\mu=0$

$$
\begin{aligned}
& I_{1}=2 t E-r \dot{r}, \\
& I_{2}=t^{2} E-t r \dot{r}+\frac{1}{2} r^{2} ;
\end{aligned}
$$

$\mu \neq 0$

$$
\begin{aligned}
& I_{1}^{\prime}=\left(\frac{1}{\mu} E-r \dot{r}+\mu r^{2}\right) e^{2 \mu t} \\
& I_{2}^{\prime}=\left(\frac{1}{\mu} E+r \dot{r}+\mu r^{2}\right) e^{-2 \mu t}
\end{aligned}
$$


In a Hamiltonian Kepler - Ermakov system the Ermakov invariant (54) is constructed $^{19}$ from the Hamiltonian and Noether invariants (58),(59) as follows:

$$
J=E^{2}-I_{1}^{\prime} I_{2}^{\prime}
$$

Finally in ${ }^{19}$ it is shown that the Ermakov invariant is generated by a dynamical Noether symmetry of the Lagrangian (52), a result which is also confirmed in. ${ }^{34}$

\section{Generalizing the Kepler-Ermakov System}

In the defining characteristics of the Kepler - Ermakov system we distinguish three different properties of reduced generality: The property of having a first integral; the property of admitting Lie/Noether symmetries with $\operatorname{sl}(2, R)$ invariance and the property of being Hamiltonian and admitting $s l(2, R)$ invariance via Noether symmetries.

We first generalize the Kepler - Ermakov system to higher dimensions by the requirement:

The generalized (Euclidian!) Kepler Ermakov system admits the sl $(2, R)$ algebra as a Lie symmetry algebra.

\subsection{The 3-d Euclidian Kepler Ermakov system}

Depending on the value $\mu \neq 0$ or $\mu=0$ we consider the three dimensional Hamiltonian Kepler Ermakov systems of type I and type II.

\subsubsection{The 3-d Hamiltonian Kepler Ermakov system of type $I(\mu \neq 0)$}

For $\mu \neq 0$ the admitted Noether symmetries are required to be (see (50)):

$$
X^{1}=\partial_{t}, X_{ \pm}=\frac{1}{\mu} e^{ \pm 2 \mu t} \partial_{t} \pm e^{ \pm 2 \mu t} R \partial_{R} .
$$

From table 6 , line 2 of ${ }^{18}$ we find that for these vectors the potential is $V(R, \phi, \theta)=-\frac{\mu^{2}}{2} R^{2}+\frac{1}{R^{2}} f(\theta, \phi)$ hence the Lagrangian is

$$
L=\frac{1}{2}\left(\dot{R}^{2}+R^{2} \dot{\phi}^{2}+R^{2} \sin ^{2} \phi \dot{\theta}^{2}\right)+\frac{\mu^{2}}{2} R^{2}-\frac{1}{R^{2}} f(\theta, \phi) .
$$

The equations of motion, that is, the equations defining the generalized dynamical system are

$$
\begin{array}{r}
\ddot{R}-R \dot{\phi}^{2}-R \sin ^{2} \phi \dot{\theta}^{2}-\mu^{2} R-\frac{2}{R^{3}} f=0, \\
\ddot{\phi}+\frac{2}{R} \dot{R} \dot{\phi}-\sin \phi \cos \phi \dot{\theta}^{2}+\frac{1}{R^{4}} f_{, \phi}=0, \\
\ddot{\theta}+\frac{2}{R} \dot{R} \dot{\theta}+\cot \phi \dot{\theta} \dot{\phi}+\frac{1}{R^{4} \sin ^{2} \phi} f_{, \theta}=0 .
\end{array}
$$


The Noether integrals corresponding to the Noether vectors are

$$
\begin{aligned}
E & =\frac{1}{2}\left(\dot{R}^{2}+R^{2} \dot{\phi}^{2}+R^{2} \sin ^{2} \phi \dot{\theta}^{2}\right)-\frac{\mu^{2}}{2} R^{2}+\frac{1}{R^{2}} f(\theta, \phi), \\
I_{+} & =\frac{1}{\mu} e^{2 \mu t} E-e^{2 \mu t} R \dot{R}+\mu e^{2 \mu t} R^{2}, \\
I_{-} & =\frac{1}{\mu} e^{-2 \mu t} E+e^{-2 \mu t} R \dot{R}+\mu e^{-2 \mu t} R^{2},
\end{aligned}
$$

where $E$ is the Hamiltonian. The Noether integrals $I_{ \pm}$are time dependent. Following $^{19}$ we consider the time independent combined first integral

$$
J=E^{2}-I_{+} I_{-}=R^{4} \dot{\phi}^{2}+R^{4} \sin ^{2} \phi \dot{\theta}^{2}+2 f(\theta, \phi) .
$$

Using (68) the equation of motion (62) becomes

$$
\ddot{R}-\mu^{2} R=\frac{J}{R^{3}}
$$

which is the autonomous Ermakov- Pinney equation. Therefore $J$ is the Ermakov invariant. $^{30}$

An alternative way to construct the Ermakov invariant (68) is to use dynamical Noether symmetries. ${ }^{35}$ Indeed one can show that the Lagrangian (61) admits the dynamical Noether symmetry $X_{D}=K_{j}^{i} \dot{x}^{j} \partial_{i}$ where $K_{i j}$ is a Killing tensor of the second rank whose non-vanishing components are $K_{\phi \phi}=R^{4}, K_{\theta \theta}=R^{4} \sin ^{2} \phi$. The dynamical Noether symmetry vector is $X_{D}=R^{2}\left(\dot{\phi} \partial_{\phi}+\dot{\theta} \partial_{\theta}\right)$ with gauge function $2 f(\theta, \phi)$.

\subsubsection{The 3-d Hamiltonian Kepler Ermakov system of type II $(\mu=0)$}

For $\mu=0$, the Noether symmetries are required to be (see $(51)):^{30}$

$$
X^{1}=\partial_{t}, X^{2}=2 t \partial_{t}+R \partial_{R}, X^{3}=t^{2} \partial_{t}+t R \partial_{R} .
$$

From table 5 , line 3 and table 6 , line 2 of $^{18}$ we find that the potential is $V(R, \phi, \theta)=$ $\frac{1}{R^{2}} f(\theta, \phi)$ hence the Lagrangian is

$$
L^{\prime}=\frac{1}{2}\left(\dot{R}^{2}+R^{2} \dot{\phi}^{2}+R^{2} \sin ^{2} \phi \dot{\theta}^{2}\right)-\frac{1}{R^{2}} f(\theta, \phi) .
$$

The equations of motion are (62) - (64) with $\mu=0$. The Noether invariants of the Lagrangian (71) are

$$
\begin{aligned}
E^{\prime} & =\frac{1}{2}\left(\dot{R}^{2}+R^{2} \dot{\phi}^{2}+R^{2} \sin ^{2} \phi \dot{\theta}^{2}\right)+\frac{1}{R^{2}} f(\theta, \phi), \\
I_{1} & =2 t E^{\prime}-R \dot{R}, \\
I_{2} & =t^{2} E^{\prime}-t R \dot{R}+\frac{1}{2} R^{2} .
\end{aligned}
$$


From the time dependent first integrals $I_{1,2}$ we define the time independent first integral $J=4 I_{2} E^{\prime}-I_{1}^{2}$ which is calculated to be

$$
J^{\prime}=R^{4} \dot{\phi}^{2}+R^{4} \sin ^{2} \phi \dot{\theta}^{2}+2 f(\theta, \phi) .
$$

Using (75) the equation of motion for $R(t)$ becomes $\ddot{R}-\frac{J^{\prime}}{R^{3}}=0$ i.e. the one dimensional Ermakov-Pinney equation, hence $J^{\prime}$ is the Ermakov invariant. ${ }^{30}$ As was the case with the 3-d Hamiltonian Kepler - Ermakov system of type I, the Lagrangian (71) admits the dynamical Noether symmetry $X_{D}=R^{2}\left(\dot{\phi} \partial_{\phi}+\dot{\theta} \partial_{\theta}\right)$ whose integral is the $J^{\prime}$.

The 3-d Hamiltonian Euclidian Kepler - Ermakov systems defined by the Lagrangians (61) and (71) need three independent first integral in involution in order to be Liouville integrable. As we have shown, each system has the two Noether integrals $(E, J)$ and $\left(E^{\prime}, J^{\prime}\right)$ respectively, hence we need one more Noether symmetry. Such a symmetry exists only for special forms of the arbitrary function $f(\theta, \phi)$. These functions and other material relevant to the study of the integrability if the 3-d Hamiltonian Euclidian Kepler - Ermakov system are given in ${ }^{36}$

\section{The $n$-Dimensional Riemannian Kepler-Ermakov System}

As it has been noted in section 8 that the Kepler - Ermakov system considered so far is the Euclidean Kepler - Ermakov system. In this section we make a drastic step forward and introduce the Riemannian Kepler Ermakov system of dimension $n$. The generalization we consider is based on the following definition:

Definition 10.1. The $n$-dimensional Riemannian Kepler - Ermakov system is an autonomous dynamical system which:

a. It is defined on a Riemannian space which admits a gradient HV;

b. Admits a first integral, which we name the Riemannian Ermakov first integral and it is characterized by the requirement that the corresponding equation of motion takes the form of the Ermakov Pinney equation;

c. It is invariant at least under the $\operatorname{sl}(2, R)$ algebra, which is generated by the gradient $\mathrm{HV}$ of the space.

There are two types of $n$-dimensional Riemannian Kepler - Ermakov systems. The ones which are not Hamiltonian and admit the $s l(2, R)$ algebra as Lie symmetries and the ones which are Hamiltonian and admit the $s l(2, R)$ algebra as Noether symmetries.

\subsection{The non-Hamiltonian Riemannian Kepler-Ermakov system}

Consider a $n$-dimensional Riemannian space which admits a gradient HV. It is well known that the metric of this space can always be written in the form ${ }^{37,38}$

$$
d s^{2}=d u^{2}+u^{2} h_{A B} d y^{A} d y^{B},
$$


where the Latin capital indices $A, B, .$. take the values $1, \ldots, n-1$ and $h_{A B}=$ $h_{A B}\left(y^{C}\right)$ is the generic $n-1$ metric. The gradient $\mathrm{HV}$ of the metric is the vector $H^{i}=u \partial_{u}(\psi=1)$ generated from the function $H=\frac{1}{2} u^{2}$. We note the relation

$$
h_{D A} \Gamma_{B C}^{A}=\frac{1}{2} h_{D B, C}
$$

The equations of motion $\frac{D x^{i}}{D t}=F$ of a particle moving in that space under the action of the force $F^{i}=F^{u}\left(u, y^{C}\right) \frac{\partial}{\partial u}+F^{A}\left(u, y^{C}\right) \frac{\partial}{\partial y^{A}}$ are

$$
\begin{aligned}
u^{\prime \prime}-u h_{A B} y^{\prime A} y^{B} & =F^{u}, \\
y^{\prime \prime}+\frac{2}{u} u^{\prime} y^{\prime A}+\Gamma_{B C}^{A} y^{B} y^{\prime C} & =F^{A} .
\end{aligned}
$$

Because the system is autonomous it admits the Lie point symmetry $\partial_{t}$. Using the vector $\partial_{t}$ and the gradient $\mathrm{HV}, H^{i}=u \partial_{u}$, we construct two representations of the $\operatorname{sl}(2, R)$ by means of the sets of vectors (see (60),(70))

$$
\begin{array}{ll}
\partial_{t}, 2 t \partial_{t}+u \partial_{u}, t^{2} \partial_{t}+t u \partial_{u} & (\mu=0), \\
\partial_{t}, \frac{1}{\mu} e^{ \pm 2 \mu t} \partial_{t} \pm e^{ \pm 2 \mu t} u \partial_{u} & \left(\mu^{2} \neq 0\right),
\end{array}
$$

and require that the vectors in each set be Lie symmetries of the system of equations (78) and (79). It is found ( $\mathrm{see}^{36}$ ) that the requirement of the invariance of the force under this group of symmetries demands that the force must be of the form

$$
F^{i}=\left(\mu^{2} u+\frac{1}{u^{3}} G^{u}\left(y^{C}\right)\right) \partial_{u}+\frac{1}{u^{4}} G^{A}\left(y^{C}\right) \partial_{A} .
$$

Replacing $F^{i}$ in the system of equations (78),(79) we find

$$
\begin{aligned}
u^{\prime \prime}-u h_{A B} y^{\prime A} y^{B} & =\mu^{2} u+\frac{1}{u^{3}} G^{u}, \\
y^{\prime \prime A}+\frac{2}{u} u^{\prime} y^{\prime A}+\Gamma_{B C}^{A} y^{\prime B} y^{\prime C} & =\frac{1}{u^{4}} G^{A} .
\end{aligned}
$$

Multiplying the second equation with $2 u^{4} h_{D A} y^{\prime D}$ and using (77) we have

$$
u^{4} \frac{d}{d s}\left(h_{D B} y^{\prime D} y^{\prime B}\right)+4 u^{3} h_{D A} u^{\prime} y^{\prime A} y^{\prime D}=2 G_{D} y^{\prime D}
$$

from which follows

$$
\frac{d}{d s}\left(u^{4} h_{D B} y^{\prime D} y^{\prime B}\right)=2 G_{D} y^{D}
$$

The first integral is

$$
J=u^{4} h_{D B} y^{\prime D} y^{\prime B}+2 \Sigma\left(y^{C}\right) .
$$

We note that $J$ involves the arbitrary metric $h_{A B}$ and the function $\Sigma\left(y^{A}\right)$. 
Finally equations (83) and (84) become

$$
\begin{aligned}
u^{\prime \prime}-u h_{A B} y^{\prime A} y^{\prime B} & =\mu^{2} u+\frac{1}{u^{3}} G^{u}\left(y^{C}\right), \\
y^{\prime \prime A}+\frac{2}{u} u^{\prime} y^{\prime A}+\Gamma_{B C}^{A} y^{B} y^{\prime C} & =-\frac{1}{u^{4}} h^{A B} \Sigma\left(y^{C}\right)_{, B} .
\end{aligned}
$$

These are the equations defining the $n$-dimensional Riemannian Ermakov - system.

Using the first integral (87) the equation of motion (88) is written as follows

$$
u^{\prime \prime}=\mu^{2} u+\frac{\bar{G}\left(y^{C}\right)}{u^{3}}
$$

where $\bar{G}^{u}=J+G^{u}\left(y^{C}\right)-2 \Sigma\left(y^{C}\right)$. This is an equation of the Ermakov-Pinney form hence we identify (87) as the Riemannian Ermakov integral of the Riemannian Kepler Ermakov system.

\subsection{The Hamiltonian Riemannian Kepler-Ermakov system}

In this section we assume that the force is derived from the potential $V\left(u, y^{C}\right)$. Then the equations of motion follow from the Lagrangian

$$
L=\frac{1}{2}\left(u^{\prime 2}+u^{2} h_{A B} y^{\prime A} y^{B}\right)-V\left(u, y^{C}\right),
$$

where $u^{\prime}=\frac{d u}{d s}$ and $s$ is an affine parameter. The Hamiltonian is

$$
E=\frac{1}{2}\left(u^{\prime 2}+u^{2} h_{A B} y^{\prime A} y^{\prime B}\right)+V\left(u, y^{C}\right) .
$$

The equations of motion are

$$
\begin{aligned}
u^{\prime \prime}-u h_{A B} y^{A} y^{B}+V_{, u} & =0, \\
y^{\prime \prime A}+\frac{2}{u} u^{\prime} y^{\prime A}+\Gamma_{B C}^{A} y^{\prime B} y^{\prime C}+\frac{1}{u^{2}} h^{A B} V_{, B} & =0 .
\end{aligned}
$$

The demand that the Lagrangian (91) admits Noether point symmetries which are generated from the gradient HV leads to the following cases (see theorem 4.2 and for details $\operatorname{see}^{33}$ )

Case A: The Lagrangian (91) admits the Noether point symmetries (80) if the potential is of the form

$$
V\left(u, y^{C}\right)=\frac{1}{u^{2}} V\left(y^{C}\right) .
$$

The Noether integrals of these Noether point symmetries are

$$
\begin{aligned}
E_{A} & =\frac{1}{2}\left(u^{\prime 2}+u^{2} h_{A B} y^{\prime A} y^{B}\right)+\frac{1}{u^{2}} V\left(y^{C}\right), \\
I_{1} & =2 t E-u u^{\prime}, \\
I_{2} & =t^{2} E-t u u^{\prime}+\frac{1}{2} u^{2},
\end{aligned}
$$

where $E_{A}$ is the Hamiltonian. 
Case B: The Lagrangian (91) admits the Noether point symmetries (81) if the potential is of the form

$$
V\left(u, y^{c}\right)=-\frac{\mu^{2}}{2} u^{2}+\frac{1}{u^{2}} V^{\prime}\left(y^{C}\right) .
$$

The Noether integrals of these Noether point symmetries are

$$
\begin{aligned}
E_{B} & =\frac{1}{2}\left(u^{\prime 2}+u^{2} h_{A B} y^{\prime A} y^{\prime B}\right)-\frac{\mu^{2}}{2} u^{2}+\frac{1}{u^{2}} V^{\prime}\left(y^{C}\right), \\
I_{+} & =\frac{1}{\mu} e^{2 \mu t} E-e^{2 \mu t} u u^{\prime}+\mu e^{2 \mu t} u^{2}, \\
I_{-} & =\frac{1}{\mu} e^{-2 \mu t} E+e^{-2 \mu t} u u^{\prime}+\mu e^{-2 \mu t} u^{2},
\end{aligned}
$$

where $E_{B}$ is the Hamiltonian.

Using the Noether integrals we construct the Riemannian Ermakov invariant for both Case A and Case B. The Riemannian Ermakov invariant is

$$
J_{G}=u^{4} h_{D B} y^{D} y^{\prime C}+2 V^{\prime}\left(y^{C}\right) .
$$

This coincides with the invariant first integral defined in (87). We note that with the use of the first integral (103) the Hamiltonian (100) takes the form

$$
E=\frac{1}{2} u^{\prime 2}-\frac{\mu^{2}}{2} u^{2}+\frac{J}{2 u^{2}},
$$

which is the Hamiltonian for the Ermakov Pinney equation.

As it was the case with the Euclidian case of section 8, it can be shown that the Riemannian Ermakov invariant (103) is due to the existence of a dynamical Noether symmetry. ${ }^{35}$

\section{Conclusion}

The Lie and the Noether point symmetry vectors of the equations of motion of an autonomous dynamical system involved in a general Riemannian space are determined completely by the geometry of the space. More specifically, Theorem 4.1 and Theorem 4.2 show that the Lie point symmetries are elements of the special projective algebra of the space and the Noether point symmetries elements of the homothetic algebra of the space. The selection of the particular vectors for a given 'force' modulating the motion of a particular system in this space is done by means of certain compatibility conditions of a differential nature which can be easily managed. Using the above theorems we have determined all 2-d and 3-d Newtonian dynamical systems which admit Lie and Noether point symmetries, we have derived in a straightforward manner the Lie point symmetries of the heat equation and finally we have generalized the Kepler - Ermakov system in a Riemannian space of dimension $n$. 


\section{References}

1. Bluman W G and Kumei S 1989 Symmetries and Differential Equations (Springer Verlag New York)

2. Olver P J 1986 Application of Lie groups to differential equations (Springer Graduate texts in Mathematics, New York: Springer)

3. Stephani H 1989 Differential Equations: Their Solutions using Symmetry (Cambridge University Press)

4. Prince G E and Crampin M (1984) Gen Rel Grav. 16, 921 -942.

5. Aminova A V and Aminov N A 2006 Sbornic Mathematics 197951

6. Aminova A V and Aminov N A 2010 Sbornic Mathematics 201631

7. Tsamparlis M and Paliathanasis A (2010) Gen. Relativ. Gravit. 42 2957-2980; (Preprint arXiv:1101.5769)

8. Tsamparlis M and Paliathanasis A (2011) Gen. Relativ. Gravit. 431861

9. Tsamparlis M, Paliathanasis A (2010), Nonlinear Dynamics 62, 203

10. Katzin G H Levine J and Davis R W 1969 J. Math. Phys. 10617

11. Hall G S and Roy I M 1997 Gen. Relativ. Gravit. 29827

12. Barnes A. 1993, Class. Quantum Grav. 10, 1139 - 1145

13. Feroze T Mahomed F M and Qadir A (2006) Nonlinear Dynamics 4565

14. Sen T 1987 Phys. Lett. A $\mathbf{1 2 2} 327$

15. Damianou P A and Sophocleous C (1999) J. Math. Phys. 40210

16. Damianou P A and Sophocleous C (2004) Nonlinear Dynamics 363

17. Tsamparlis M. and Paliathanasis A (2011) J. Phys. A: Math. and Theor. 44 175202; (Preprint arxiv:1205.4114)

18. Tsamparlis M and Paliathanasis A (2012) J. Phys. A: Math and Theor. 275201; (Preprint arXiv:1111.0810)

19. Moyo S and Leach P G L 2002 J. Phys. A: Math and Gen 355333

20. Haas F and Goedert J 2001 Phys. Lett. A 279181

21. Athorne C 1991 Phys. Lett. A 159375

22. Govinder K S AthorneC Leach P G L 1993 J. Phys. A: Math and Gen 264035

23. Govinder K S Leach P G L 1993 Quaestiones Mathematicae 16405

24. Ermakov V 1880 Univ. Isz. Kiev Series III 9 (translated by Harin A O)

25. Leach P G L and Andriopoulos K 2008 Appl. Anal. Discrete Math. 2146

26. Rogers C Hoenselaers C and Ray J R 1993 J. Phys. A: Math. and Gen. 262625

27. Schief W K Rogerts C and Bassom A P 1996 J. Phys. A: Math. and Gen. 29903

28. Goedert J and Haas F. 1998 Phys. Lett. A 239348

29. Athorne C 1991 J. Phys. A: Math and Gen 24 L1385

30. Leach P G L 1991 Phys. Lett. A 158102

31. Govinder K S and Leach P G L 1974 Phys. Lett. A 186391

32. Leach P G L and Karasu A 2004 J. Nonlinear Math. Phys. 11269

33. Tsamparlis M and Paliathanasis A 2011 J. Phys. A: Math. and Theor. 44175202

34. Haas F and Goedert J 1996 J. Phys. A: Math. Gen. 294083

35. Kalotas T M and Wybourne B G 1982 J. Phys A: Math and Gen 152077

36. Tsamparlis M and Paliathanasis A (2012) J. Phys. A: Math and Theor. 275202

37. Colley A A Tupper B O J 1989 J. Math. Phys 3011

38. Colley A A Tupper B O J 1990 J. Math. Phys 313 